\title{
INTEGRAL All-Sky Survey: Deep inside Galaxy and beyond
}

\author{
R. Krivonos ${ }^{a b *}$, M. Revnivtsev ${ }^{b c}$, S. Tsygankov ${ }^{a b}$, S. Sazonov ${ }^{b}, \mathbf{A}$. Vikhlinin $^{b d}$, \\ M. Pavlinsky ${ }^{b}$,E. Churazov ${ }^{a b}$ and R. Sunyaev ${ }^{a b}$ \\ ${ }^{a}$ Max-Planck-Institute für Astrophysik, \\ Karl-Schwarzschild-Str. 1, D-85740 Garching bei München,Germany \\ ${ }^{b}$ Space Research Institute, Russian Academy of Sciences, \\ Profsoyuznaya 84/32, 117997 Moscow, Russia \\ ${ }^{c}$ Excellence Universe Cluster, Munich Technical University, \\ Boltzman-Str. 2, D-85748 Garching bei München, Germany \\ ${ }^{d}$ Harvard-Smithsonian Center for Astrophysics, \\ 60 Garden Street, Cambridge, MA 02138, USA
}

\begin{abstract}
This report is related to a hard X-ray survey covering the whole sky performed with the INTEGRAL observatory during seven years. We present the improved method for image reconstruction with IBIS coded mask telescope. The main improvements are related to suppression of systematic effects which strongly limit sensitivity in the region of the Galactic Plane (GP), especially in the crowded field of the Galactic Center (GC). We extended the IBIS/ISGRI background model to take into account Galactic Ridge X-ray Emission (GRXE). To suppress residual systematic artifacts on the reconstructed sky image we apply nonparametric sky image filtering based on wavelet decomposition. The implemented modifications of the sky reconstruction method decreases the systematic noise by $\sim 44 \%$ in the region of the GC and practically removes it from high-latitude sky images, leaving sensitivity in the only dependence from Poisson statistics. This allows one to conduct all-sky survey with a highest available at the moment sensitivity in Galactic Plane: $3.7 \times 10^{-12} \mathrm{erg} \mathrm{s}^{-1} \mathrm{~cm}^{-2} \sim 0.26 \mathrm{mCrab}$ at a $5 \sigma$ detection level in the $17-60 \mathrm{keV}$ working energy band. The survey covers $90 \%$ of the sky down to flux limit of $6.2 \times 10^{-11} \mathrm{erg} \mathrm{s}^{-1} \mathrm{~cm}^{-2}(\sim 4.32 \mathrm{mCrab})$ and $10 \%$ of the sky area down to flux limit of $8.6 \times 10^{-12} \mathrm{erg} \mathrm{s}^{-1} \mathrm{~cm}^{-2}(\sim 0.60 \mathrm{mCrab})$.
\end{abstract}

The Extreme sky: Sampling the Universe above $10 \mathrm{keV}$ - extremesky2009,

October 13-17, 2009

Otranto (Lecce) Italy

\footnotetext{
*Speaker.

†E-mail: krivonos@mpa-garching.mpg.de
} 


\section{Introduction}

INTEGRAL observatory [1] gathered a huge observational data set allowing to perform the most sensitive hard X-ray survey up to date. The main scientific results and source catalogs are reported in many relevant papers $[2,3,4,5,6,7,8,9,10,11,12,13]$.

Recently, the great progress in surveying hard X-ray sky was achieved with Burst Alert Telescope [14] onboard Swift observatory [15]. The Swift/BAT survey provides very homogeneous sky coverage in 15-195 keV energy band with current maximum sensitivity $2.2 \times 10^{-11} \mathrm{erg} \mathrm{s}^{-1} \mathrm{~cm}^{-2}$, peaked in extragalactic sky. The survey results and source catalogues are reported in papers [16] and [17]. As seen from large sample of detected Active Galactic Nuclei (AGNs), the results of Swift/BAT survey are very high value for extragalactic studies. The sky coverage of Swift/BAT is nearly uniform, therefore only small fraction of its total operational time is devoted to observations of the Galaxy.

Contrary to Swift, INTEGRAL observatory provides all-sky survey with sensitivity more concentrated in Galactic Plane, having typical limiting flux less than $1.43 \times 10^{-11} \mathrm{erg} \mathrm{s}^{-1} \mathrm{~cm}^{-2}(1$ mCrab) in working energy range 17-60 keV. This makes Swift/BAT and INTEGRAL surveys complementary to each other.

INTEGRAL accumulated already a lot of exposure time in the direction of the Galactic plane (with maximum $\sim 20 \mathrm{Msec}$ of nominal time in the direction of the Galactic Center). However, at the moment the growing exposure time devoted to the plane and the Center of the Galaxy is not reflected in corresponding increase of the survey sensitivity. Observations in these regions are strongly affected by systematics related to crowded field of GC and strong Galactic X-ray background radiation. The top panel of Fig. 1 demonstrate reconstructed sky of the central part of the Galaxy. The underlying sky background is not flat as it should be for coded-aperture imaging. Strong systematic artifacts mimic extended structures on the sky at high detection significance level $(5-10 \sigma)$, increase background noise, and prevent automatic source detection. Among main sources of systematic noise in GC observations we emphasize the following:

1. The response of IBIS telescope to the point source is not modeled properly. This leads to small fraction of source flux being unaccounted. In the case of bright source observation, the systematic residuals can be strong.

2. The extended X-ray emission of the Galaxy ("Ridge") significantly affects background illumination of the ISGRI detector, and makes the simple background considerations wrong.

3. Due to periodicity of IBIS mask pattern, the very significant side peaks ("ghosts") appears in the point spread function. The proximity of source position to the "ghost" position of another source causes ambiguity in flux determination of both sources. Appearence of many bright sources in FOV strengthens the self-influence of sources in flux determination procedure.

In our related paper [18] we describe methods later refered as general sky reconstruction algorithm. These methods provide basic IBIS/ISGRI data analysis developed by one of us (EC), and used in previous papers $[19,3,20,10]$. In the following sections we discuss the mentioned sources of systematic noise and present several improvements for IBIS/ISGRI sky reconstruction method. 

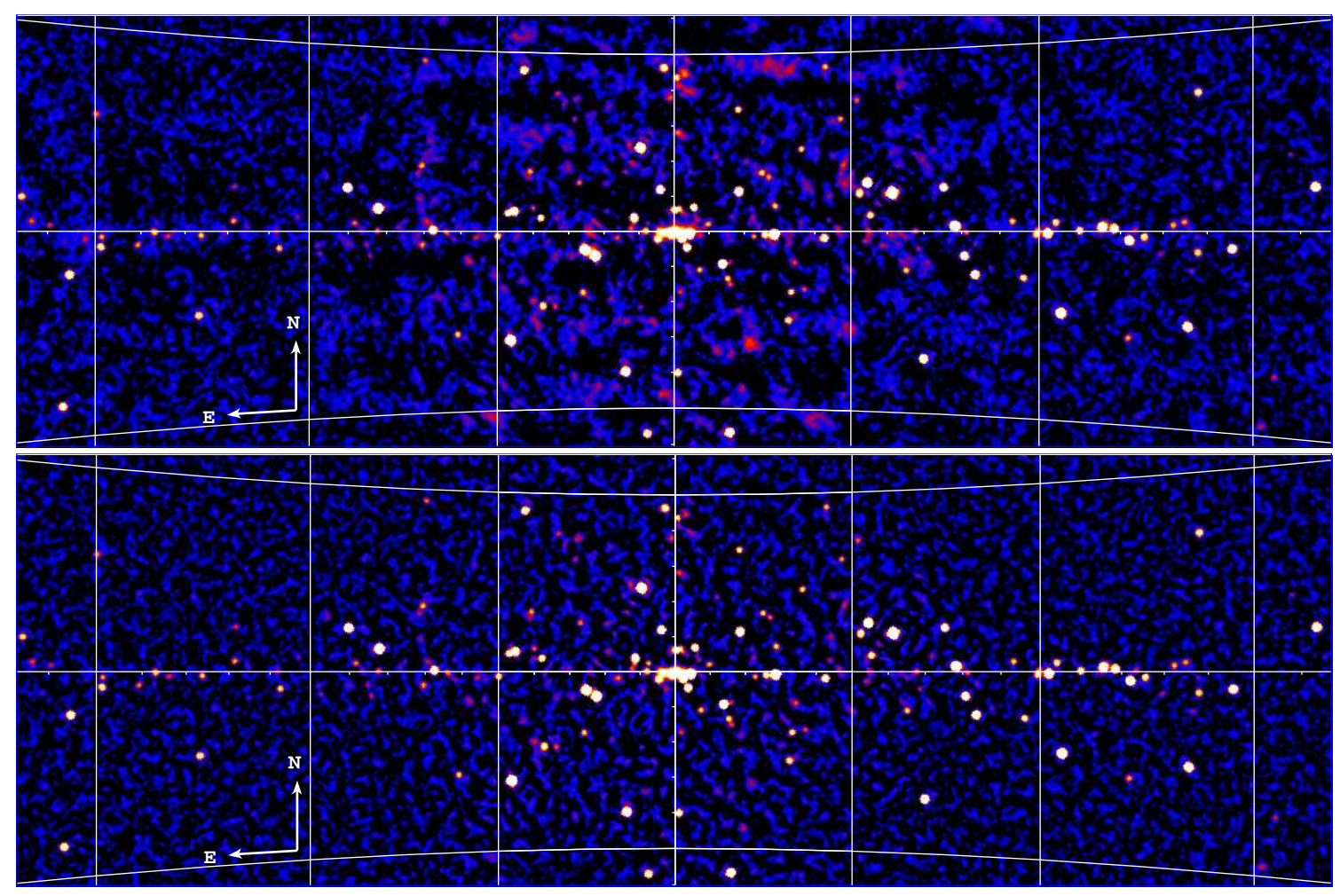

Figure 1: Map of the sky region near the Galactic plane obtained with IBIS/ISGRI in the $17-60 \mathrm{keV}$ energy band. The total exposure is about $20 \mathrm{Ms}$ in the region of Galactic Center. The images are shown in significances with squared root color scale ranging from 0 to 25 . Such color scheme is used to emphasize sky background variations. The black and blue color correspond to pixel values from 0 to 2 . The red pixels have values around 5. The yellow to white color transition corresponds to 15 and more. Upper panel: sky mosaic provided by general sky reconstruction method. Lower panel: sky mosaic produced by improved reconstruction algorithm with taking into account Galactic X-ray Background and removing systematic artifacts on sky images with wavelet transform.

\section{Source projection model}

IBIS is coded aperture imaging telescope [21]. The sky is projected to the detector plane through transparent/opaque elements of the mask mounted above detector plane. Generally, the sky reconstruction is based on deconvolution of the detector image with known mask pattern.

The quality of reconstructed sky image directly depends on our understanding of coding procedure. The IBIS telescope coding procedure of incoming photons is not only the function of mask transparency. For example, the mask supporting structure, can significantly reduce number of low energy photons passed though the open mask elements. The different montage elements of the IBIS mask block the incoming photons, modifying the detector image created by a point source (shadowgram). We have implemented the best known to date configuration of all known elements of the telescope, which make contribution to the shadowgram. The parameters of these elements were approximately found by comparison of actual and model detector shadowgrams illuminated by strong source at different azimuthal angles. 

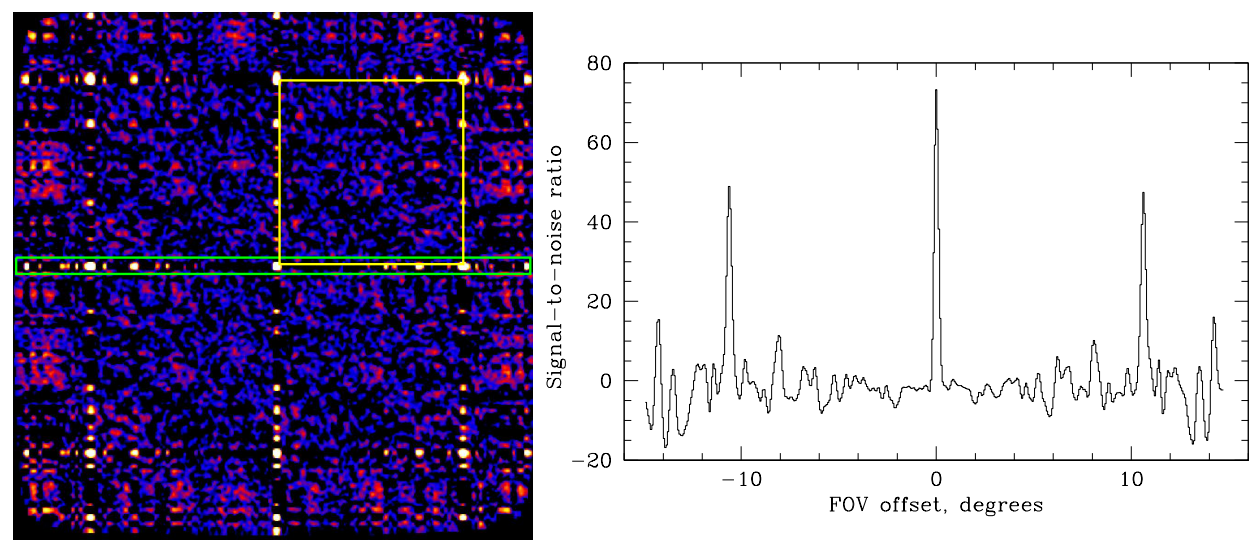

Figure 2: Right: Sky image of bright source Crab Nebula centered on axis. Periodic mask pattern leads to many false sources on reconstructed sky. The most significant false peaks are located in vertexes of a $\sim 10.5^{\circ}$ square around source, oriented in detector coordinates as illustrated by yellow square. Left: Image profile extracted from $1^{\circ} \times 29^{\circ}$ green region. The profile demonstrates response of the IBIS/ISGRI imaging system to the point source.

Additional complication arises due to fact that the IBIS mask has replicated patterns [21]. This pattern of the mask have some advantage because in ideal case it has much narrower point spread function and flat sidelobes in the central part of the reconstructed image [22]. But at the same time it causes serious complications due to presence of very significant side peaks of the point spread function. It means that simple deconvolution algorithm sees "ghost" sources at certain sky positions (Fig. 2), related to the position of the real source and the size of the replicated mask pattern.

The presence of significant side peaks of the PSF means that sources in a variety of sky positions within a field of view create shadows with similar patterns, which causes self-influence of sources and uncertainty of its flux determination. Unfortunately, this situation is not rare in the crowded field of Galactic Center, as shown on Fig. 3.

In order to obtain a good quality all-sky map, suitable for search of new weak sources, the ghosts of known bright sources have to be removed. Since the pattern of the shadowgram cast by a point source through the mask is not ideally known, the source removal procedure is not perfect. Some photon counts might be left or oversubtracted at specific positions on the detector. This effect is usually small but can become significant for deep Galactic Center observations and fields containing very bright sources. For the last, characteristic "crosses" and "rings" appear around the bright sources (see Fig. 4). The former artifact appears when the observational program of a bright source is dominated by observations with a constant roll angle. A variable roll angle diminishes this effect but produces concentric structures. The level at which imperfect ghost removal starts to play a role depends on the observational pattern and typically corresponds to $\sim(3-5) \times 10^{-3}$ of the flux of the brightest source within the IBIS field of view. For example, systematic residuals around Crab reach $\sim 10$ mCrab flux $(17-60 \mathrm{keV})$, near Sco X-1 $\sim 5-7$ mCrab, and GC region contains artifacts with flux $\sim 0.5-1 \mathrm{mCrab}$. 


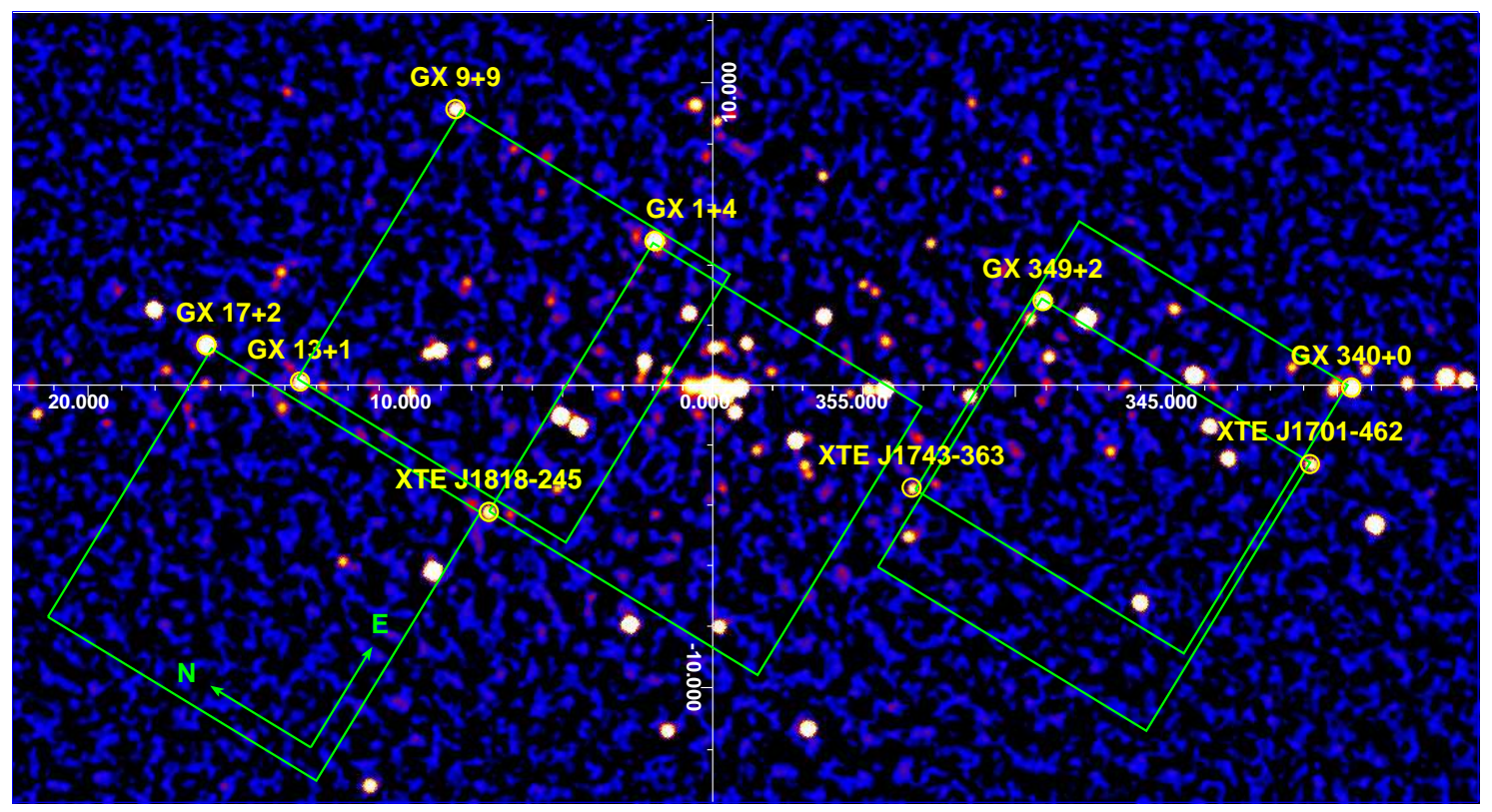

Figure 3: Zoom in map of the sky region around Galactic Center. The blue squares demonstrate relative sky positions of false side peaks ("ghosts") of selected bright sources (see Fig. 2). Due to observational constrains IBIS FOV was mainly co-aligned with axes of equatorial coordinate system ("N-E" notation). As a result, there are a number of bright sources which permanently appeared in mutual "ghost" positions. This fact leads to source self-influence in flux extraction procedure. The following source pairs can be affected by mutual flux interplay: GX 340+0 and XTE J1743-363, XTE J1701-462 and GX 349+2, GX $9+9$ and GX $13+1$, and triplet GX 1+4, XTE $J 1818-245$ and GX $17+2$.

\section{Galactic X-ray Background}

From early X-ray observations we know, that Galaxy reveals itself as a strong diffuse emitter [23]. The morphology of Galactic X-ray background at energies above $20 \mathrm{keV}$ is now relatively well known. As shown in RXTE and INTEGRAL investigations [24, 20], X-ray background is traced by near infrared brightness of the Galaxy, having components of Galactic disk and Bulge.

The presence of Galactic X-ray Background in the IBIS FOV strongly affects the overall shape of the ISGRI detector shadowgram, which leads to significant systematic artifacts on reconstructed sky images. According to our estimations, Ridge emission introduces $\sim 40 \%$ of total systematic noise for source-free field on $\sim 300 \mathrm{ksec}$ exposure. We extended IBIS/ISGRI background model to take into account Galactic Ridge X-ray Emission [18].

\section{Sources self-influence in Galactic Center}

By summing up all shadowgrams of bright sources in GC observations, we show [18] that practically all detector pixels are illuminated by at least one source. It means, that any measurements of source fluxes in GC will be affected by correlations between sources, especially for that ones having replicated patterns (see Fig. 3). This fact leads to source self-influence in flux extraction procedure. The situation is further complicated by the fact, that practically all ISGRI pixels are illuminated at the same time by the Ridge emission. As a result, the correlation arises between 

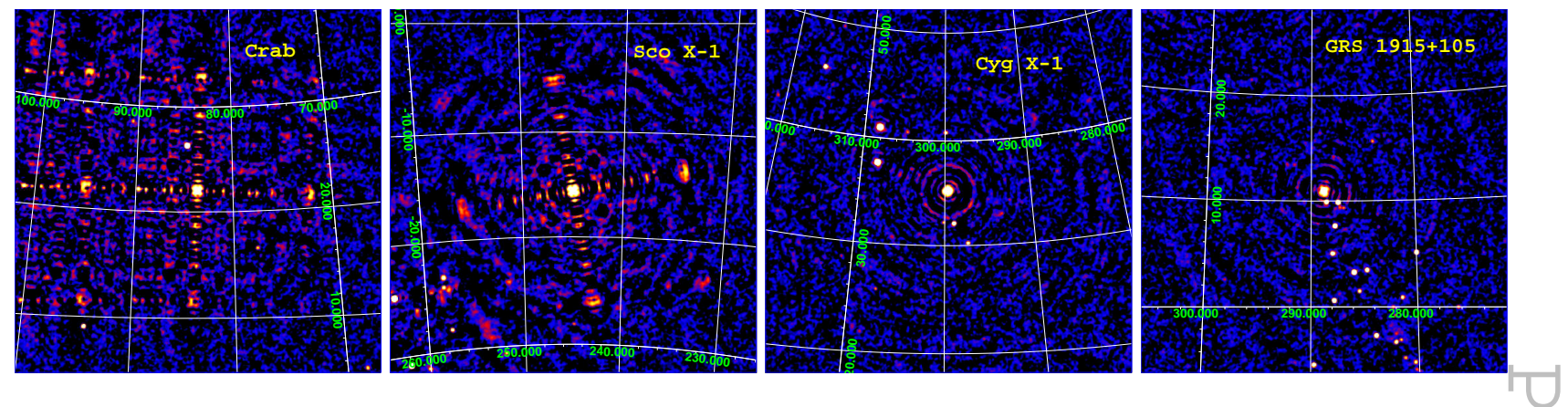

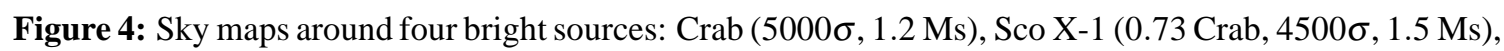

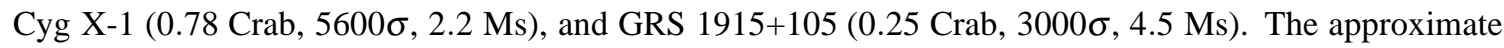
$17-60 \mathrm{keV}$ flux in units of Crab flux, detection significance, and dead-time corrected exposure on each source, respectively, are indicated in brackets.

shadowgrams of a manifold of sources and the Ridge. In order to disentangle the Ridge detector component from source shadowgrams we use different spacecraft orientations relative to Galactic Plane. To do this, we simultaneously process nearest observations before and after the given one. As a result, undesired self-influence of sources and Ridge emission is significantly reduced. For details, we refer reader to [18].

\section{Noise reduction on sky images}

To reduce systematic noise on sky images we use spatial frequency information. As seen from deep staring observations of NGC 4151 on Fig. 5, systematic residuals introduce background variations at some angular scale (characteristic chessboard-like squares and ripples), which is apparently different from spatial scale of the point sources. This allows us to construct image filtering procedure based on wavelet decomposition. The key point of all wavelet methods is that the wavelet transform (WT) is able to discriminate structures as a function of spatial scale. In the context of individual IBIS/ISGRI observation, we are interested in removing all large scale structures from non-uniform sky background. The task is greatly simplified by the fact, that coded-mask aperture technique is not able to reconstruct image of objects with spatial size greater then angular resolution of telescope. Therefore, all structures on image, more extended than point source are artificial and can be surely removed. By other words, we do not need in any threshold clipping to discriminate noise and signal, we can remove systematic residuals with a given angular scale. In this way, the WT works as non-parametric (model-free) background approximation. To decompose sky image, we use à trous digital wavelet transform algorithm [25].

We performed WT decomposition and filtering of sky images of NGC 4151 staring mode campaign. It is clearly seen from Fig. 5 that all systematic artifacts are totally removed from the image, leaving clean sky image with unit standard deviation as expected for pure Poisson statistics.

\section{Results and conclusions}

By taking into account Galactic X-ray Background and using WT sky filtering we obtain new deep image of Galactic Plane which is demonstrated on the lower panel of Fig.1. As seen from 

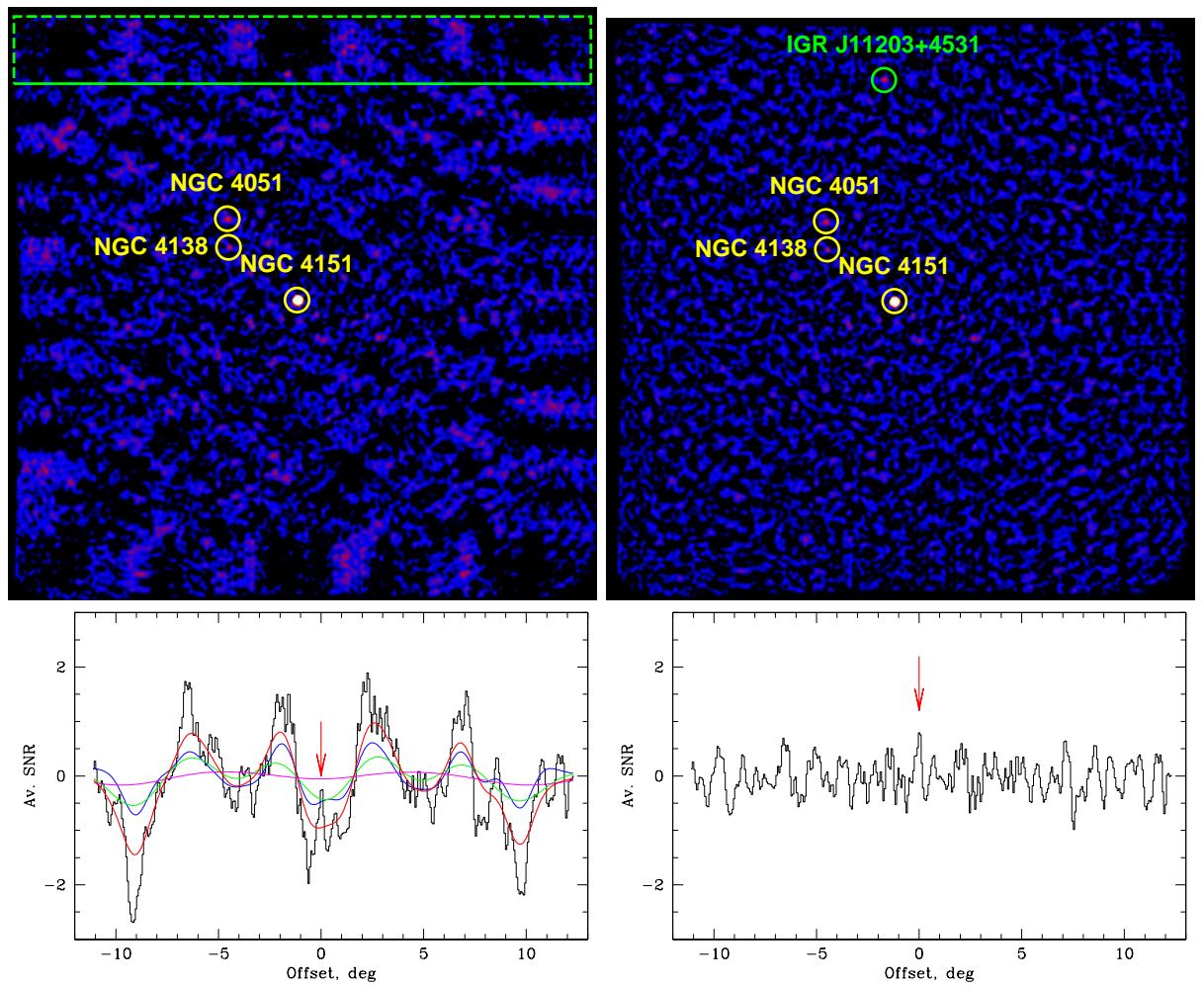

Figure 5: Top Panel: $30^{\circ} \times 30^{\circ}$ sky region around NGC 4151 accumulated with sequence of 95 staring observations (rev. 74-76). The total dead-time corrected exposure is $280 \mathrm{ksec}$. The left mosaic image is obtained by general method. Image on the right was produced by summing up images of individual ScWs corrected with WT algorithm. Standard deviation of source-free pixels on the left and right images relates as 1.3 and 1.0, respectively. Previously unknown hard X-ray source IGR J11203+4531 detected during this observation is marked by green label. Bottom Panel: Vertically averaged profiles of corresponding sky images extracted from denoted green region. The X-axis is measured in offset from IGR J11203+4531 position (marked by red arrow). Note, that source amplitude shown on this plot is averaged, for actual source detection significance see Fig. 10 in [18]. Blue, green and magenta curves are profiles of corresponding decomposition components at spatial scales $\sim 1,2$, and 4 degrees, respectively. Actually, the sum of these components (red curve) is sky image background approximation which is to be removed from original image.

the sky image most of the systematic artifacts are removed, leaving more or less uniform sky background. Obviously, the quality of reconstructed sky is improved. To estimate the efficiency of improved reconstruction method, we measured the standard deviation of image pixels masking out bright sources. For cleaned and general sky standard deviation relates as $1.33 / 1.84$, which gives us the total sensitivity improvement by $\sim 28 \%$. Assuming pure statistics having unit standard deviation, the suppression of systematic noise in GC region is $\sim 44 \%$.

Most of the INTEGRAL observing time was spent in Galactic Plane and Center, giving us possibility to conduct the most sensitive survey of Milky Way above $20 \mathrm{keV}$ ever made. The minimal detectable flux with $5 \sigma$ detection level reached the level of $3.7 \times 10^{-12} \mathrm{erg} \mathrm{s}^{-1} \mathrm{~cm}^{-2}$, which is $\sim 0.26 \mathrm{mCrab}$ in $17-60 \mathrm{keV}$ energy band. The survey covers $90 \%$ of the sky down to flux limit of $6.2 \times 10^{-11} \mathrm{erg} \mathrm{s}^{-1} \mathrm{~cm}^{-2}(\sim 4.32 \mathrm{mCrab})$ and $10 \%$ of the sky area down to flux limit of $8.6 \times 10^{-12} \mathrm{erg} \mathrm{s}^{-1} \mathrm{~cm}^{-2}(\sim 0.60 \mathrm{mCrab})$. 
We conclude, that the implemented modifications of IBIS/ISRGI sky reconstruction method significantly decreases the systematic noise in the region of Galactic Center and practically removes it from high-latitude observations. This allows to more efficiently reflect the growing sky exposure into the sensitivity of INTEGRAL survey.

\section{Acknowledgments}

This work was supported by grants of the RFBR (08-02-00974, 09-02-00867, NSH-5069.2010.2) and programs of Presidium of RAS P19 and OFN-16. SS acknowledges the support of the Dynasty Foundation.

\section{References}

[1] Winkler C., et al., 2003, A\&A, 411, L1

[2] Revnivtsev, M. G., Sazonov, S. Y., Gilfanov M. R., Sunyaev, R. A. 2003a, Astronomy Letters, 29, 587

[3] Krivonos, R., Vikhlinin, A., Churazov, E., et al., 2005, ApJ, 625, 89

[4] Revnivtsev, M. G., Sazonov, S. Y., Molkov, S. V., et al., 2006, Astronomy Letters, 32, 145

[5] Bird, A. J., et al. 2004, ApJ, 607, L33

[6] Bird, A. J., et al. 2006, ApJ, 636, 765

[7] Bird, A. J., et al. 2007, ApJS, 170, 175

[8] Bassani, L., et al. 2006, ApJ, 636, L65

[9] Bazzano, A., et al. 2006, ApJ, 649, L9

[10] Krivonos, R., Revnivtsev, M., Lutovinov, A., et al., 2007, A\&A, 475, 775

[11] Sazonov, S., Revnivtsev, M., Krivonos, R., Churazov, E., \& Sunyaev, R. 2007, A\&A, 462, 57

[12] Beckmann, V., et al. 2009, arXiv:0907.0654

[13] Bird, A. J., et al. 2009, arXiv:0910.1704

[14] Barthelmy, S. D., et al. 2005, Space Science Reviews, 120, 143

[15] Gehrels, N., et al., 2004, ApJ, 611, 1005

[16] Tueller, J., et al. 2009, arXiv:0903.3037

[17] Cusumano, G. 2009, American Institute of Physics Conference Series, 1126, 104

[18] Krivonos, R., et al., 2010, INTEGRAL/IBIS 7-year All-Sky Hard X-Ray Survey. Part I: Image Reconstruction. submitted to A\&A.

[19] Revnivtsev, M., Sunyaev, R., Varshalovich, D., et al. 2004, Astronomy Letters, 30, 382

[20] Krivonos, R., Revnivtsev, M., Churazov, E., et al., 2007, A\&A, 463, 957

[21] Ubertini P., et al., 2003, A\&A, 411, L131

[22] Fenimore, E.E., Cannon, T.M.: 1978, APO, 17, 337

[23] Worrall D. M., Marshall F. E., Boldt E. A., Swank J. H., 1982, ApJ, 255, 111

[24] Revnivtsev, M., Sazonov, S., Gilfanov, M., Churazov, E., \& Sunyaev, R. 2006, A\&A, 452, 169

[25] Vikhlinin, A., Forman, W., \& Jones, C. 1997, ApJ, 474, L7 